Author: Marek Krok

Title of article: „MindSphere V2 do obrabiarek sterowanych numerycznie” ("MindSphere V2 for CNC machine tools”)

Mechanik, Vol. 91, No. 8-9 (2018): pages 658-660

DOI: https://doi.org/10.17814/mechanik.2018.8-9.103

\title{
MindSphere V2 for CNC machine tools
}

\section{MindSphere V2 do obrabiarek sterowanych numerycznie}

\section{MAREK KROK*}

Presented is the concept of the implementation of the Internet of Things (IoT) using the MindSphere platform offered by Siemens with accompanying additional hardware and software dedicated to numerically controlled machine tools.

KEYWORDS: Internet of Things, CNC machine tools, data acquisition, data analysis

The Internet of Things (loT) offers many new opportunities both in the private sphere as well as in the industry and service sector. IoT is about connecting the real world with the virtual world of data. Devices can send information to the cloud for processing, or you can manage and control them using dedicated applications. This scenario becomes a reality thanks to more and more miniaturized computers, affordable sensors, a ubiquitous computer network and the growing availability of smart devices in many areas of the industry and beyond.

Siemens has expanded the concept of the loT with industrial applications. The presented case concerns machine tools with SINUMERIK Operate control.

\section{MindSphere}

The MindSphere cloud solution is an open architecture server application, created in response to the needs of the industrial sector. This platform allows the implementation of digitalization processes in the organization by connecting devices, machines and plant infrastructure to it. It provides a data collection service and computing power to process them to generate added value for the enterprise. Thanks to advanced analytics, MindSphere provides information (including on the use and possibilities of machine park optimization), supports the planning of periodic inspections of devices, provides effective prediction of failures, and allows to reduce energy consumption in the enterprise [1].

MindSphere is maintained by Siemens, which enables even small production companies to collect data that will optimize the operation of CNC machine tools and will significantly increase the productivity of processes in which such machines are used.

The MindConnect subassemblies, used to acquire information from machine tools, use security mechanisms that encrypt and send data only to the MindSphere platform, identifying the appropriate security certificate. Verified certificates and keys used by MindConnect Elements are a necessary condition for the correct operation of the platform, which will not accept data from an unauthorized device, and

* Mgr inż. Marek Krok (marek.krok@siemens.com) - RC-PL DF MC Siemens in the case of pharming attacks MindConnect Element will block sending information out of the plant until the problem is solved.

Siemens offers the MindSphere loT operating system in the form of a platform as a service (PaaS) based on the cloud. It allows customers to create their own applications, run them and manage them without having to build their own infrastructure or services related to managing software packages.

The view of the MindSphere V2 basic screen with default applications is shown in fig. 1 .

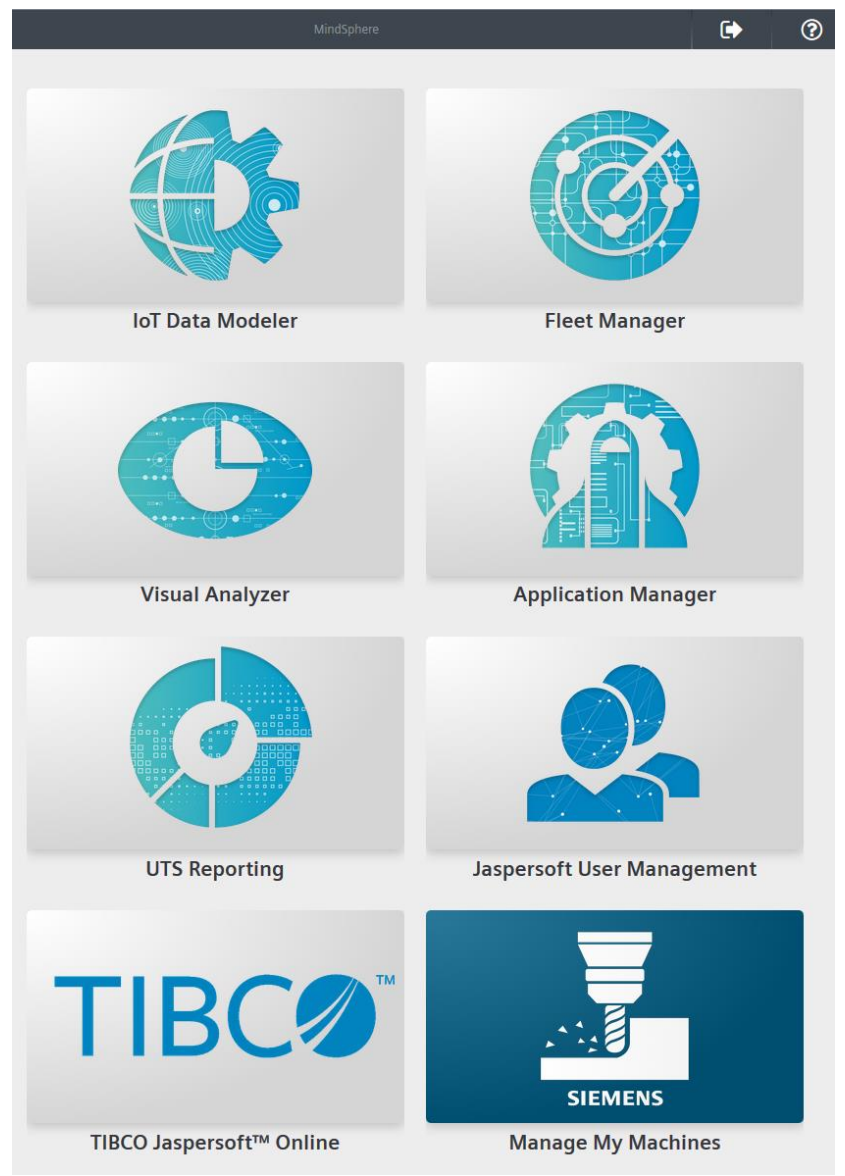

Fig. 1. MindSphere V2 main screen

\section{Standard applications}

MindSphere provides a number of applications that allow you to manage the platform and support data acquisition and interpretation. 
The standard application IoT Data Modeler provides the basic functionality of MindSphere support for users, organization structure and its resources.

There are two levels of access for users: an administrator and a standard user. The administrator has the authority to create the organization structure in the form of suborganization and departments within a given structure. You can create new resources in your organization that represent devices that are numerically controlled machine tools in this case. The next task of the administrator is to create new users and assign them to the created organizational structures. On the other hand, in the case of IoT Data Modeler, users can view the created resources and prepare orders for downloading data (the so-called aspects) from the machine tool.

Fleet Manager is a standard application for analysis, visualization and ongoing monitoring of device status based on resources created in IoT Data Modeler. Fleet Manager uses devices and aspects defined in loT Data Modeler. It offers the following functions: monitoring the current status of devices in the context of acquisition status (online/offline), representation of devices deployed around the world, visualization of data of a specific type on the preview page, manual creation of service tasks, preparation of rules for automatically creating service rules and e-mail notifications based on the current value of a given aspect or group of aspects [3].

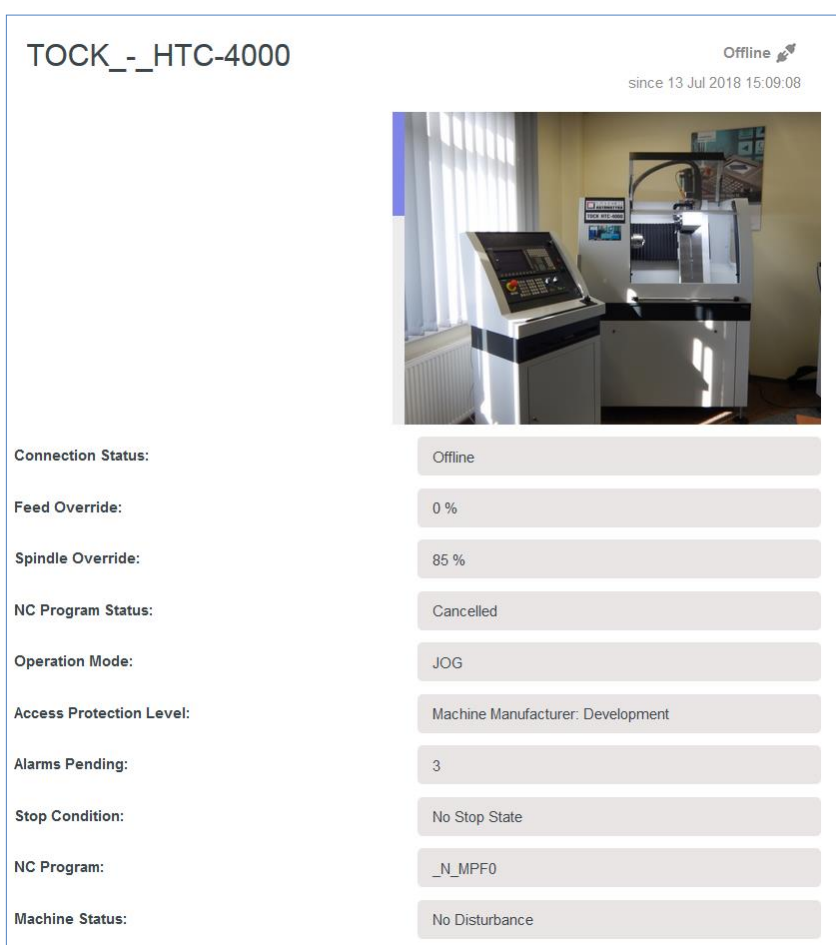

Fig. 2. Manager MyMachines application screen for numerically controlled machine tools

Visual Analyzer is the basic tool for visualizing the state of resources configured in the MindSphere application. Where Fleet Manager is a tool for device management, Visual Analyzer is a tool for accurate viewing of data with the possibility of creating specific views of registered variables, as well as creating and exporting charts and sharing them with colleagues.

The Manage MyMachines application is designed for machine tools equipped with SINUMERIK control. Offers an overview of the machine tools configured in MindSphere and displaying relevant information about their condition. The parameter package for SINUMERIK 840D sl and SINUMERIK 828D control is registered and displayed on the Manage MyMachines dashboard. You can make the previously defined data points dependent on system events to automatically display this information. The view of the parameter screen for the Manage MyMachines application is shown in fig. 2.

\section{Data collection methods}

There are several possibilities to integrate devices, including CNC machine tools with the MindSphere system [2].

MindConnect Nano - allows you to connect the IPC 227E NanoBox device to collect data via various protocols (Siemens S7 and OPC UA Data Access). This device supports the transmission of encrypted data via a secure internet connection. The unit has a data read cycle of up to 250 data points per second and goes through a data transfer cycle every 10 seconds. These parameters allow for continuous monitoring of even a complicated industrial process, and at the same time provide insight, almost in real time, to the monitored machine.

MindConnect IOT 2040 - allows to receive data through the same protocols as MindConnect Nano, but the amount of data sent to MindSphere is limited to 30 points per second. This frequency is intended for a less extensive production environment or a single machine tool. An exemplary view of the installed MindConnect loT 2040 with the SINUMERIK 828D controller is shown in fig. 3.

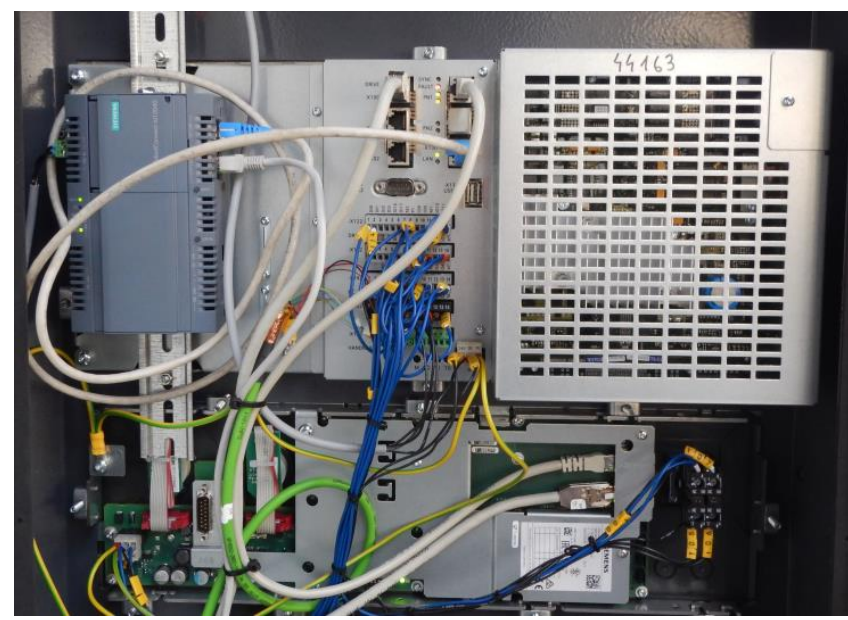

Fig. 3. MindConnect loT 2040 installed with the SINUMERIK 828D controller

Both MindConnect Nano and MindConnect loT 2040 use the OPC UA protocol to collect information from the SINUMERIK control. OPC UA (Unified Architecture). It is a standard, industrial communication protocol providing access to process data, e.g. by control systems. Variables can be read and written in SINUMERIK 840D sl and SINUMERIK 828D using this communication protocol with the SINUMERIK Integrate Access MyMachine / OPC UA software option. The OPC UA server has been integrated with SINUMERIK 840D sI and SINUMERIK 828D from the software version 4.5 SP3. Access is possible, for example, for CNC, PLC, GUD, MD data and setting data. Communication with the OPC UA server takes place on a standard Ethernet industrial network protocol. It is also possible to collect information from other controls equipped with an OPC UA server.

MindConnect FB 1500 - allows you to connect the S7-1500 driver to MindSphere without additional hardware.

MindConnect Lib - is a program library that provides API (Application Programming Interface) to build your own devices and programs that allow communication with MindSphere.

SINUMERIK Integrate Client - is a system application built into the SINUMERIK Operate control desk that allows direct transfer of data to MindSphere (SINUMERIK 840D sI 
from version 4.5 SP4 HF1 and SINUMERIK 828D from version $4.5 \mathrm{SP} 4 \mathrm{HF} 6$ ), however, to ensure the stability of the production process, the number of data transmitted is limited up to 50 data points for 5 seconds.

\section{Data processing and analysis}

With a view to processing and analyzing data in MindSphere, the TIBCO Jaspersoft ${ }^{\mathrm{TM}}$ service has been implemented, consisting of a solution based on the local Jaspersoft Studio ${ }^{\mathrm{TM}}$ application and on the Jaspersoft ${ }^{\mathrm{TM}}$ Online cloud.

The online tool, i.e. the TIBCO Jaspersoft ${ }^{\mathrm{TM}}$ service, enables the visualization of data by creating individual reports and views. Due to these visualizations, one can find hidden connections of process data and identify the potential for optimization. The screen view of the TIBCO Jaspersoft ${ }^{\mathrm{TM}}$ online application is shown in fig. 4.

The Jaspersoft Studio ${ }^{\mathrm{TM}}$ PC application allows to prepare report templates, panels and visualizations, as well as to connect to data sources other than MindSphere in the company, for example to the ordering system, quality control system or storage and tool management. Due to this, it allows to find more complex correlations than would be possible only on the basis of process data.

Application Manager is a system application. It is necessary for creating and downloading templates for writing, registering and adding applications to the MindSphere Launchpad portal when developing and using own applications written specifically for a specific MindSphere user.

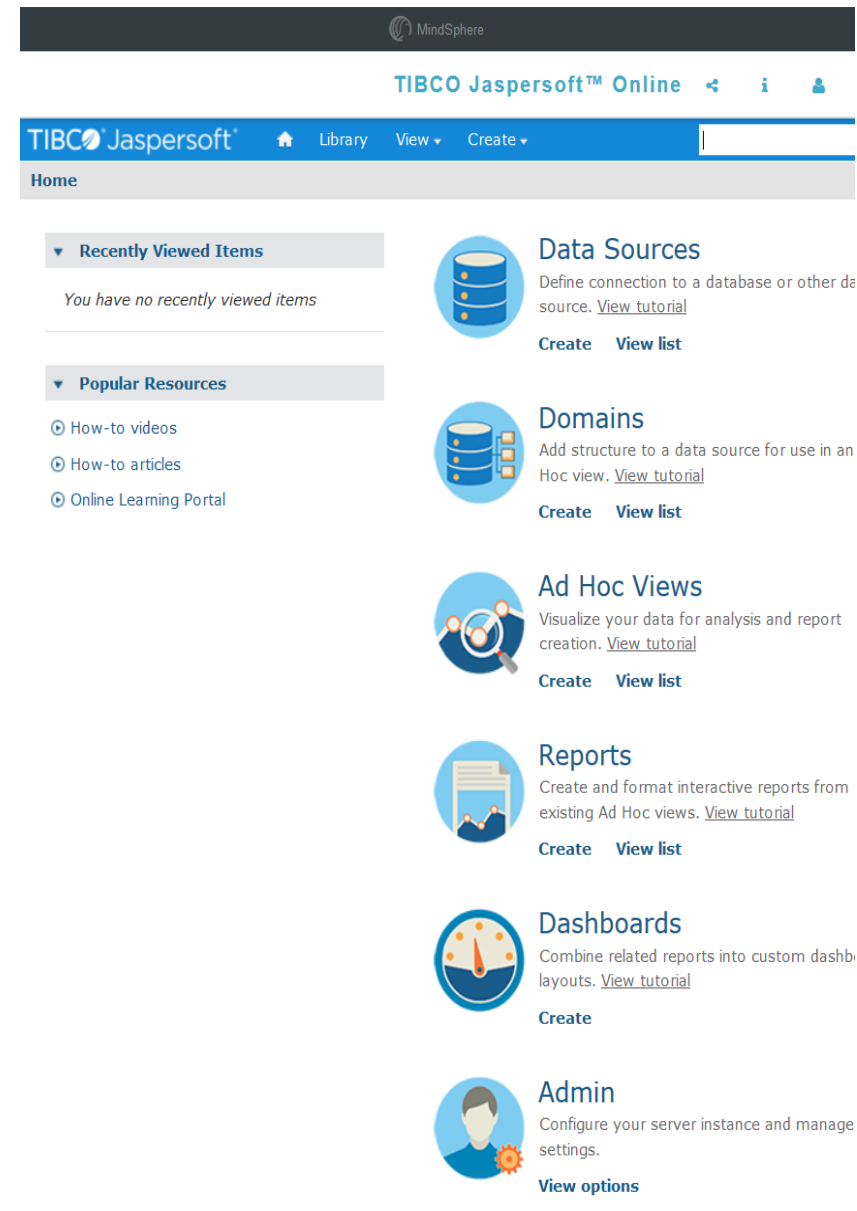

Fig. 4. Screen of the TIBCO Jaspersoft ${ }^{\mathrm{TM}}$ application online

\section{Implementations in Poland}

In order to present the capabilities of the MindSphere system and to the needs of the MachTool 2018, a test installation was prepared. It includes three training centers (PCKPiEZ in Łańcut, EngSolution in Bielsko-Biała, Siemens Training Center in Bielsko-Biała) and test machines from three manufacturers (DMG MORI/Famot, AFM Defun, AVIA). To show the functionalities of the system, all the possibilities of connection to the MindSphere application for numerically controlled machine tools were applied.

\section{Conclusions}

MindSphere application, due to advanced analytics, provides the user with a lot of information previously unavailable, including on the use of the machine park and the possibilities of its optimization, it supports the planning of periodic inspections of devices, provides effective prediction of failures, and also helps reduce the energy consumption in the enterprise. The constantly developing MindSphere platform is supplemented with new functionalities.

Currently, Siemens is migrating the developed solutions to MindSphere V3, which provides even greater reliability and a lot of new possibilities, and in combination with custom-developed applications, allows to meet all user expectations.

\section{REFERENCES}

1. „Getting Connected to MindSphere”. Industry Support Online. ID: $109755908(05 / 2018)$

2. "MindSphere - Getting Started (MindConnect Nano \& IoT2040)". Industry Support Online. ID: 109483499 (10/2017).

3. „MindSphere - Fleet Manager - System Manual”. Industry Support Online. ID: 109749651 (09/2017).

4. „SINUMERIK 840Dsl/828D Integrate Access MyMachine/OPC UA”. Industry Support Online. ID: 109757507 (04/2018).

Translation of scientific articles, their computer composition and publishing them on the website www.mechanik.media.pl by original articles in Polish is a task financed from the funds of the Ministry of Science and Higher Education designated for dissemination of science. 